\title{
Détermination du zéro hydrographique dans un système de référence terrestre mondial
}

\author{
Arnaud Ballay $^{1}$, Bernard Simon ${ }^{2}$ et Guy Wöppelmann ${ }^{3}$ \\ ${ }^{1}$ Doctorant, Université de Caen / EPSHOM, 13 rue du Chatellier, BP 30316, \\ 29603 Brest cedex, (33)2.98.22.15.75 ballay@shom.fr \\ ${ }^{2}$ Ingénieur sur contrat, EPSHOM, (33)2.98.22.18.68 simon@shom.fr \\ 3 Enseignant chercheur, CDLG, Université de La Rochelle, avenue Michel \\ Crépeau, 17042 La Rochelle cedex 1, (33)5.46.45.86.13 gwoppelm@univ-lr.fr
}

Résumé. L'Organisation Hydrographique Internationale (OHI) recommande que le Niveau des Plus Basses Mers Astronomiques soit adopté comme le zéro hydrographique international. Après avoir adopté cette recommandation, au Service Hydrographique et Océanographique de la Marine (SHOM), une nouvelle approche a été développée pour déterminer le zéro hydrographique et pour réduire les sondages bathymétriques. Cette approche est basée sur des techniques spatiales incluant le GPS, la modélisation du niveau des plus basses mers et du niveau moyen de la mer. Ainsi le zéro résultant peut être considéré comme relié à un système géodésique de référence global bien défini et maintenu comme l'ITRF (International Terrestrial Reference Frame).

\begin{abstract}
The International Hydrographic Organisation recommends that Lowest Astronomical Tide (LAT) to be adopted as the international Chart Datum. Many hydrographic offices, like SHOM in France, have now adopted this recommendation. A new approach to fix chart datum and rectify bathymetric surveys depth measurements will be presented. It is based on modern space techniques, including GPS, lowest astronomical tide calculation and mean sea level ellipsoidal heights. This subsequent chart datum can then be considered as heights related to a well defined and maintained global geodetic reference frame like ITRF.
\end{abstract}

\section{Introduction}

Les données marégraphiques ont été historiquement utilisées, et le sont toujours, pour définir et réaliser des systèmes de référence verticale maritimes et terrestres, notamment le niveau moyen et le zéro hydrographique. Ce zéro hydrographique sert de référence pour les profondeurs indiquées sur les cartes marines et aussi pour les prédictions de la marée. Depuis 1996, l'Organisation Hydrographique Internationale (OHI) recommande que le Niveau des Plus Basses Mers astronomiques (NPBM) soit adopté comme le zéro hydrographique international. Cette référence est définie comme le plus bas niveau de la mer sous des conditions météorologiques moyennes résultant de la combinaison de différentes conditions astronomiques. C'est un niveau théorique sous lequel la mer ne descend que très rarement. Ainsi le navigateur est presque toujours sûr d'avoir au moins la hauteur d'eau indiquée sur sa carte marine. Néanmoins les réalisations pratiques du zéro hydrographique dévient souvent de la définition 
théorique. Les différences viennent principalement des limites techniques qui sont sources d'erreurs lors des réductions des sondages bathymétriques au zéro hydrographique. Les variations locales de cette surface le long de la côte dépendent du marnage et des fluctuations spatiales et temporelles de la surface de la mer. Actuellement dans le cadre de sondages bathymétriques, on localise le zéro hydrographique grâce à :

-un marégraphe temporaire proche de la zone d'étude, -un marégraphe permanent le plus proche sur la côte, -un modèle de marée pour tenir compte des variations de la marée loin des marégraphes.

La procédure actuellement utilisée implique les hypothèses suivantes:

- la pente du niveau moyen entre les marégraphes temporaires et permanents est constante,

- l'action de la météorologie est la même sur toute la zone,

- Le modèle fournit des résultats précis.

Les imprécisions viennent du fait que ces hypothèses ne sont pas toujours vérifiées.

La méthode actuelle de réduction des sondages est basée sur la formule harmonique de la marée qui suppose que l'on connaisse pour avoir une bonne précision, l'amplitude et la phase initiale d'environ une centaine de composantes harmoniques de la marée. Le niveau moyen de la mer est la moyenne des hauteurs de marée, rapportée au niveau des plus basses mers ; c'est le terme constant de la formule harmonique. En se servant de marégraphes, on peut connaître les constantes harmoniques et ainsi corriger les influences météorologiques. Les zéros hydrographiques actuels en France sont basés sur des données marégraphiques. Pour les ports de référence, les observations continues sur plusieurs années d'un marégraphe permanent et des repères locaux permettent une bonne définition du zéro hydrographique; pour les marégraphes temporaires, le zéro est basé sur moins d'un an d'observations du niveau de la mer (un mois en général) et est déterminé par concordance avec le port de référence le plus proche. Pour ces observatoires temporaires, il est donc difficile de déterminer avec précision le niveau moyen et par conséquent le niveau des plus basses mers.

Considérant les éléments exposés ci-dessus, l'Organisation Hydrographique Internationale (OHI) a recommandé que la référence verticale internationale pour le zéro hydrographique soit un ellipsoïde d'un repère terrestre. L'Association Internationale de Géodésie (résolution de 1'AIG, Vienne 1991) recommande que pour la géodésie, la géodynamique ou l'océanographie de haute précision, l'ITRS (International Terrestrial Reference System) ou un système rattaché soit utilisé. Le WGS84 ne devrait être utilisé que lorsqu'une précision submétrique n'est pas nécessaire.

Nous présenterons dans cet article une nouvelle méthode pour déterminer le zéro hydrographique et corriger les sondages bathymétriques, basée sur des données d'altimétrie spatiale, la modélisation numérique et de positionnement. 
Dans la dernière partie, nous présenterons les premiers résultats obtenus avec cette approche dans le Golfe de Gascogne et dans la Manche.

\section{L'approche spatiale}

\subsection{Principe}

Les techniques de géodésie spatiale comme GPS, DORIS, VLBI et SLR fournissent des jeux de coordonnées en trois dimensions des stations qui sont combinées pour réaliser l'ITRS et l'ITRF (Boucher [1]). Les coordonnées verticales sont référencées à l'ITRS. On utilise les données des satellites altimétriques (ERS1, ERS2 ou TOPEX-POSEIDON) pour déterminer la différence entre la surface océanique moyenne (temporelle) et l'ellipsoïde de l'ITRS ce qui fournit des surfaces moyennes océaniques (SMO). Les données marégraphiques et les modèles hydrodynamiques fournissent le niveau marin sur les sites côtiers et au large. L'analyse de ces données permet d'obtenir la fonction de base pour calculer et prédire les niveaux de la mer, le niveau des plus basses mers inclus.

Le GPS en mode statique différentiel est utilisé pour connecter entre eux les marégraphes de référence et donne leur position verticale dans l'ITRS. Les niveaux marins peuvent être ainsi référencés dans l'ITRS et cela permet de comparer, d'évaluer et d'améliorer la SMO à la côte où l'altimétrie spatiale est moins précise.

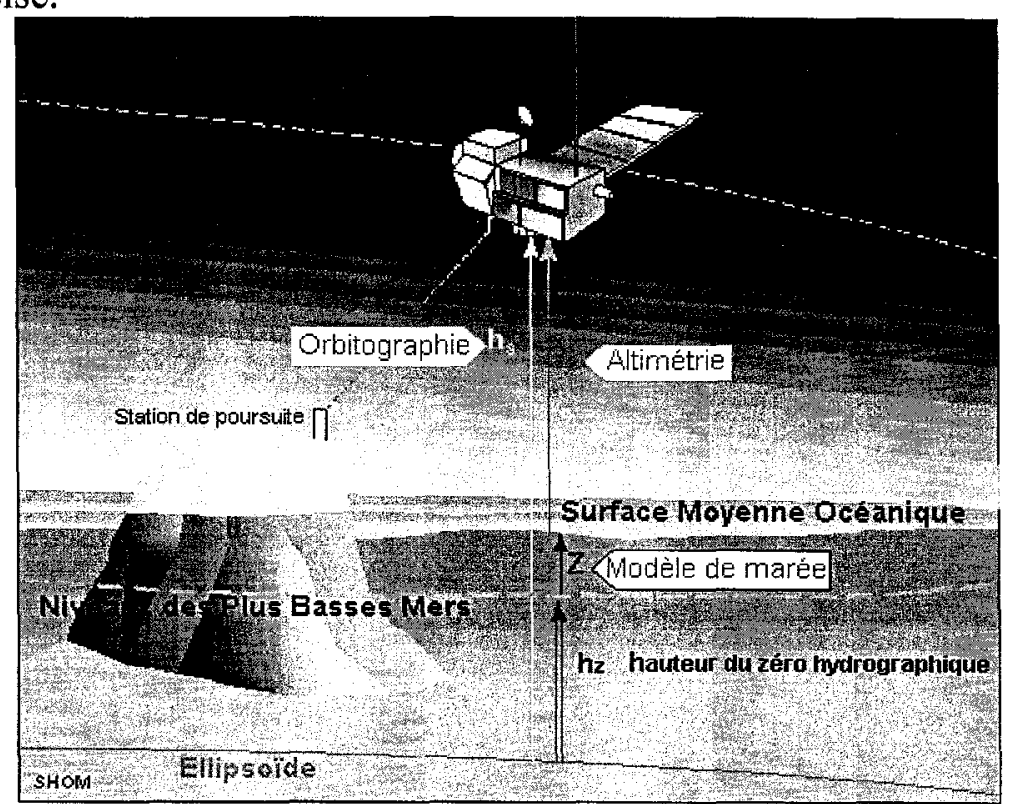

Figure 1 Approche spatiale (Wöppelmann et al [6])

Figure 1 Spatial approach (Wöppelmann et al [6])

La figure 1 illustre le principe de la méthode (Wöppelmann et al [6]) : connaissant le niveau moyen par rapport à l'ellipsoïde avec les satellites d'altimétrie $\left(h_{s}-D\right)$, et le zéro hydrographique (NPBM) par rapport au niveau moyen, on peut déduire 
le zéro par rapport à l'ellipsoïde. La relation suivante donne la hauteur du zéro hydrographique $\left(h_{z}\right)$ par rapport à l'ellipsoöde choisi : $h_{z}=\left(h_{s}-D\right)-Z$.

Le modèle de marée fournit l'écart $(Z)$ entre le niveau moyen de la mer et le niveau des plus basses mers.

\subsection{La Surface Moyenne Océanique (SMO)}

Le SHOM et la société CLS ont calculé et cartographié le niveau moyen de la mer par rapport à un ellipsoïde de référence avec une précision de quelques centimètres à partir d'une base de données altimétriques contenant dix ans de mesures. La SMO «SHOM-CLS v98.2 » (Hernandez [2]) a été calculée sur la référence fournie par le profil moyen sur 3 ans de Topex/Poséidon (T/P). Les données T/P, ERS1 et Geosat ont été fusionnées par méthode inverse basée sur une technique d'interpolation suboptimale, pour calculer les hauteurs moyennes de la mer avec une résolution de 1/16 de degré, ainsi que les erreurs associées. La surface est calculée là où les données sont valables c'est-à-dire entre $82^{\circ} \mathrm{N}$ et $82^{\circ} \mathrm{S}$. La SMO comprend les ondulations du géoïde marin ( \pm 120 mètres). La figure 2 montre la carte de la SMO. Nous verrons dans la troisième partie (figure 5) qu'à proximité de la côte la précision de la surface n'est pas très bonne. Mais en allant vers le large, la précision devient vite acceptable pour notre but.

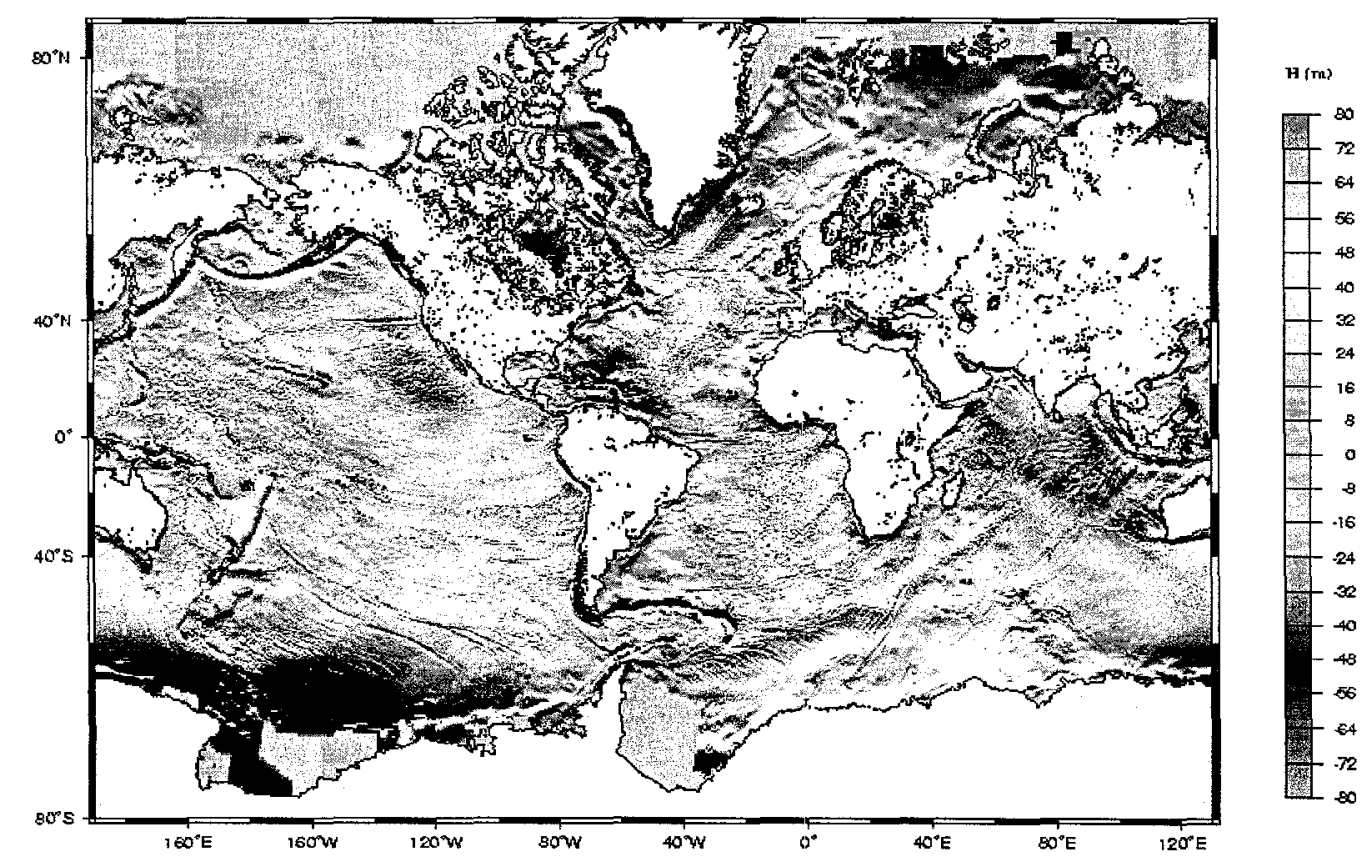

Figure 2 Surface Moyenne Océanique « SHOM-CLS v98.2 »

Figure 2 Mean Sea Surface (MSS) «SHOM-CLS v98.2》

\subsection{Les modèles de marée}

La marée astronomique a été calculée en utilisant un modèle numérique aux éléments finis. La figure 3 présente les résultats de la plus basse mer astronomique calculée à partir d'un modèle numérique (ajusté aux observations) de constantes harmoniques. 


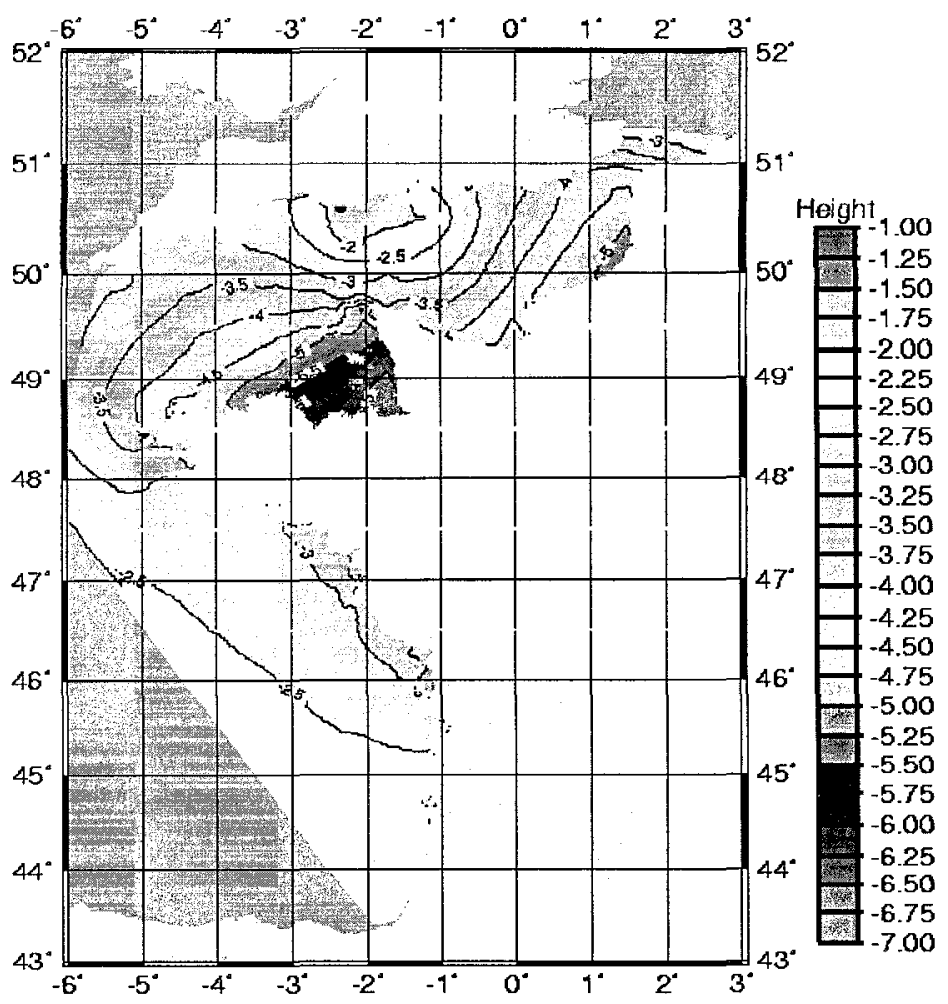

Figure 3. Modèle de marée:

$\mathrm{NPBM} /$ niveau moyen

Figure 3. Tidal model: LAT / MSL

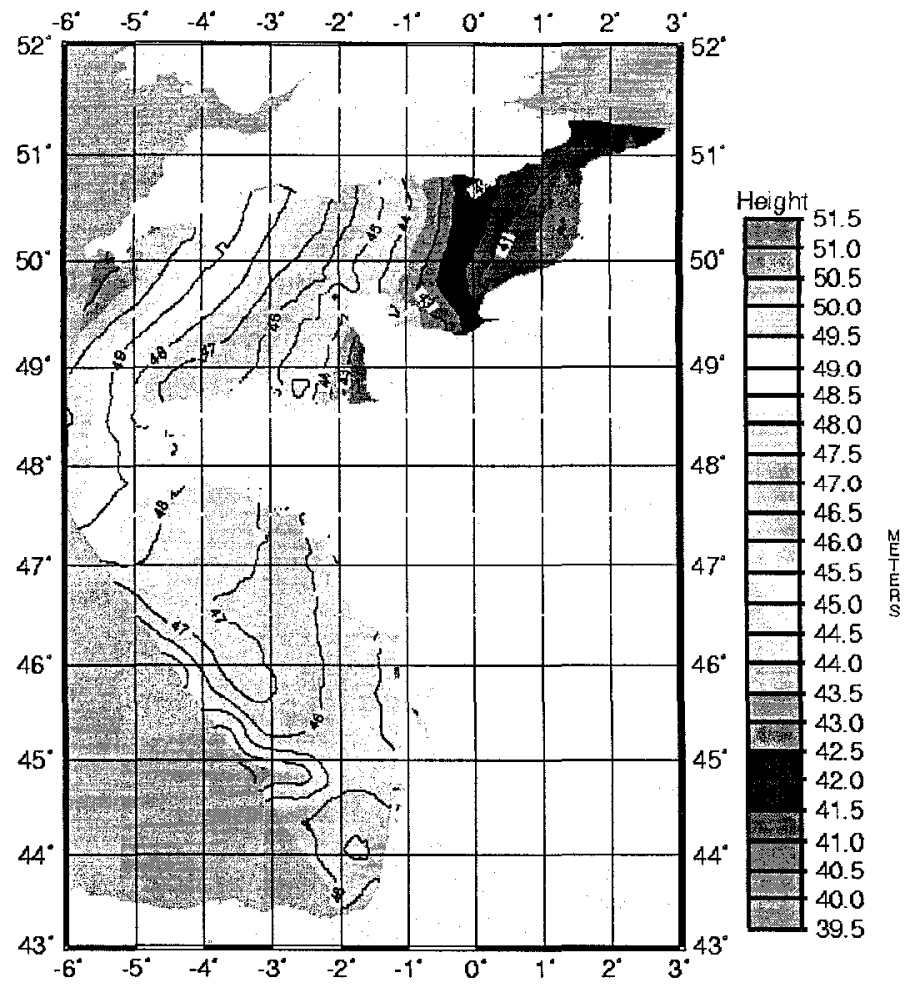

Figure 4. Réalisation du zéro hydrographique : NPBM / ellipsoïde (ITRS)

Figure 4. Chart datum realisation : LAT / ellipsoid (ITRS)

\section{Premiers résultats}

\subsection{Réalisation spatiale du zéro hydrographique}

En connaissant le niveau moyen par rapport à l'ellipsoïde fourni par la SMO et la plus basse mer astronomique par rapport au niveau moyen fourni par le modèle, on peut déduire le plus bas niveau de la mer (le zéro hydrographique) par rapport à l'ellipsoïde (figure 4).

Les différents facteurs affectant la précision de la réalisation du zéro sont :

- la précision de la surface verticale de référence (précision de l'ITRS),

- les erreurs de la SMO,

- les erreurs sur la prédiction du niveau d'eau (erreurs sur le NPBM).

Pour notre objectif, une précision de 10 centimètres sur le positionnement du zéro hydrographique dans l'ITRS serait suffisante (tableau 1).

\begin{tabular}{|c|c|c|c|}
\hline & Côte (d<25km) & $\mathbf{2 5}<\mathbf{d}<\mathbf{5 0} \mathbf{k m}$ & $\mathbf{d}>\mathbf{5 0} \mathbf{~ k m}$ \\
\hline ITRF & $2 \mathrm{~cm}$ & $2 \mathrm{~cm}$ & $2 \mathrm{~cm}$ \\
\hline SMO & $24 \mathrm{~cm}$ & $8 \mathrm{~cm}$ & $4 \mathrm{~cm}$ \\
\hline NPBM & $6 \mathrm{~cm}$ & $6 \mathrm{~cm}$ & $6 \mathrm{~cm}$ \\
\hline Total & $\mathbf{2 5} \mathrm{cm}$ & $\mathbf{1 0} \mathrm{cm}$ & $7 \mathrm{~cm}$ \\
\hline
\end{tabular}

Tableau 1: Evaluation des erreurs

Table 1. Error evaluation 
Le tableau 2 présente les résultats de la connexion de marégraphes à des GPS pour évaluer la SMO à la côte. Ils sont cohérents à $95 \%$.

\begin{tabular}{|l|c|c|c|}
\hline \multicolumn{1}{|c|}{ Station } & SMO (m) & TG + GPS (m) & |Difference \\
\hline Dunkerque & $44,54 \pm 0,21$ & $44,88 \pm 0,04$ & 0,34 \\
Dieppe & $45,59 \pm 0,23$ & $53,85 \pm 0,04$ & 0,05 \\
Le Havre & $46,21 \pm 0,24$ & $46,27 \pm 0,04$ & 0,06 \\
Le Conquet & $51,89 \pm 0,15$ & $52,08 \pm 0,03$ & 0,19 \\
Brest & $51,62 \pm 0,20$ & $51,91 \pm 0,03$ & 0,30 \\
St J. de Luz & $49,52 \pm 0,44$ & $49,86 \pm 0,02$ & 0,33 \\
Leixoes & $55,89 \pm 0,26$ & $55,77 \pm 0,05$ & 0,12 \\
Vigo & $54,35 \pm 0,09$ & $54,09 \pm 0,03$ & 0,26 \\
Lagos & $53,35 \pm 0,12$ & $53,31 \pm 0,03$ & 0,03 \\
Alicante & $50,79 \pm 0,24$ & $50,61 \pm 0,02$ & 0,19 \\
\hline
\end{tabular}

Tableau 2. Evaluation de la SMO

Table 2. (Mean sea surface evaluation)

Pour améliorer la SMO à la côte nous avons combiné les valeurs du niveau moyen des marégraphes connectés aux GPS et de la SMO en utilisant des fonctions de pondération de ces deux niveaux. Les poids attribués à la SMO sont des fonctions inverses des erreurs associées. Pour les marégraphes, la fonction de pondération dépend de la distance du point de la surface au marégraphe : elle est maximum au marégraphe et minimum dès que les erreurs de la SMO sont acceptables. Les figures 5 et 6 montrent les incertitudes associées à la SMO et les erreurs de la SMO améliorée dans la Manche.

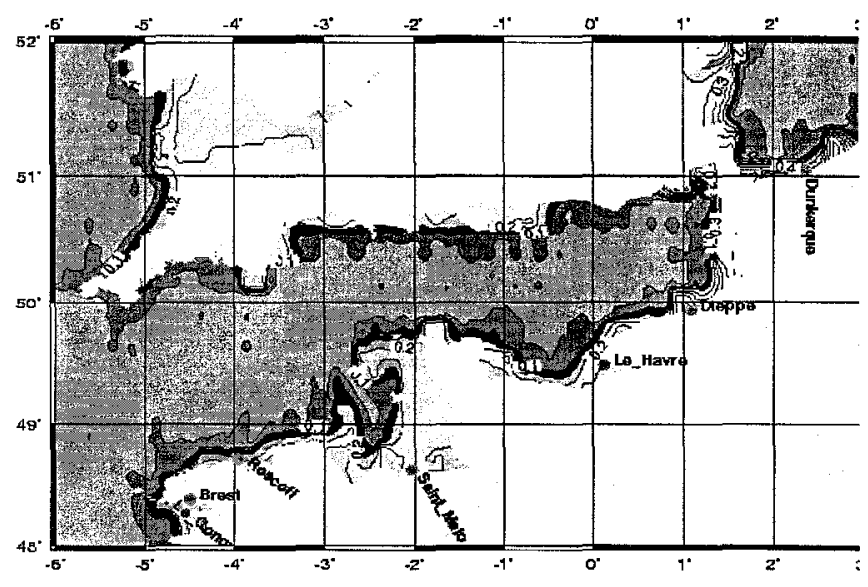

Figure 5 Erreurs de la SMO originale Figure 5 Original MSS errors

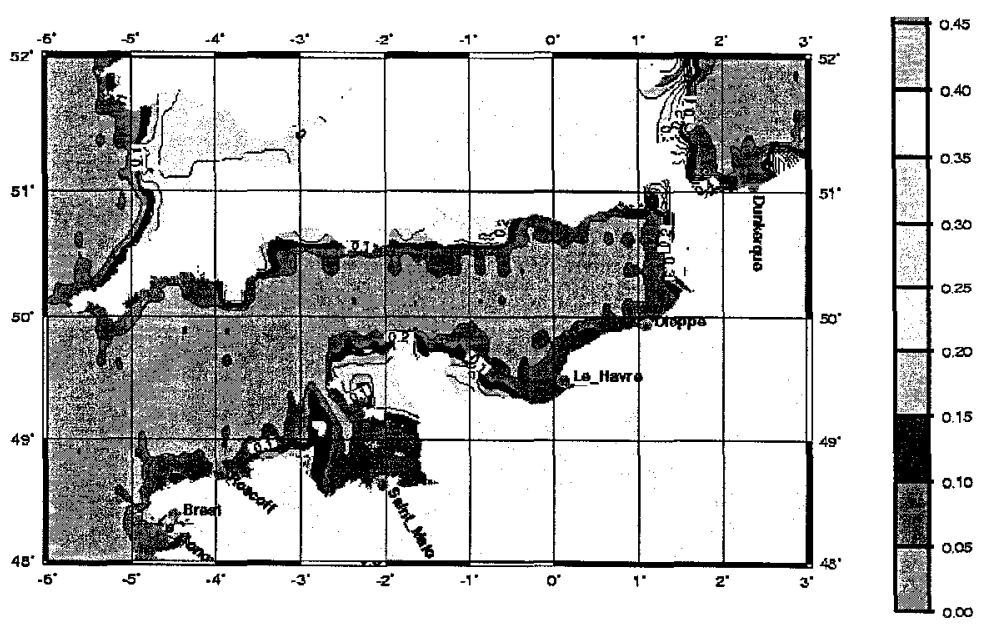

Figure 6 Erreurs de la SMO améliorée Figure 6 Improved MSS errors

\subsection{Application pour les sondages.}

Utilisé en mode cinématique, on peut se servir du GPS pour le positionnement vertical et ainsi retrouver la référence du niveau d'eau mesuré (zéro 
hydrographique). Les fonds marins sont localisés dans la même surface de référence verticale utilisée par le navigateur et les réductions aux sondages sont alors facilitées (Wöppelmann et al [6]).

Le principe de réduction des sondages est illustré dans la figure 7. En mesurant la topographie $\mathrm{T}$ des fonds marins par rapport à l'ellipsoïde et en connaissant le zéro hydrographique $\left(\mathrm{h}_{\mathrm{Z}}\right)$ par rapport au même ellipsoïde, on peut en déduire la sonde réduite $S_{\mathrm{r}}$.

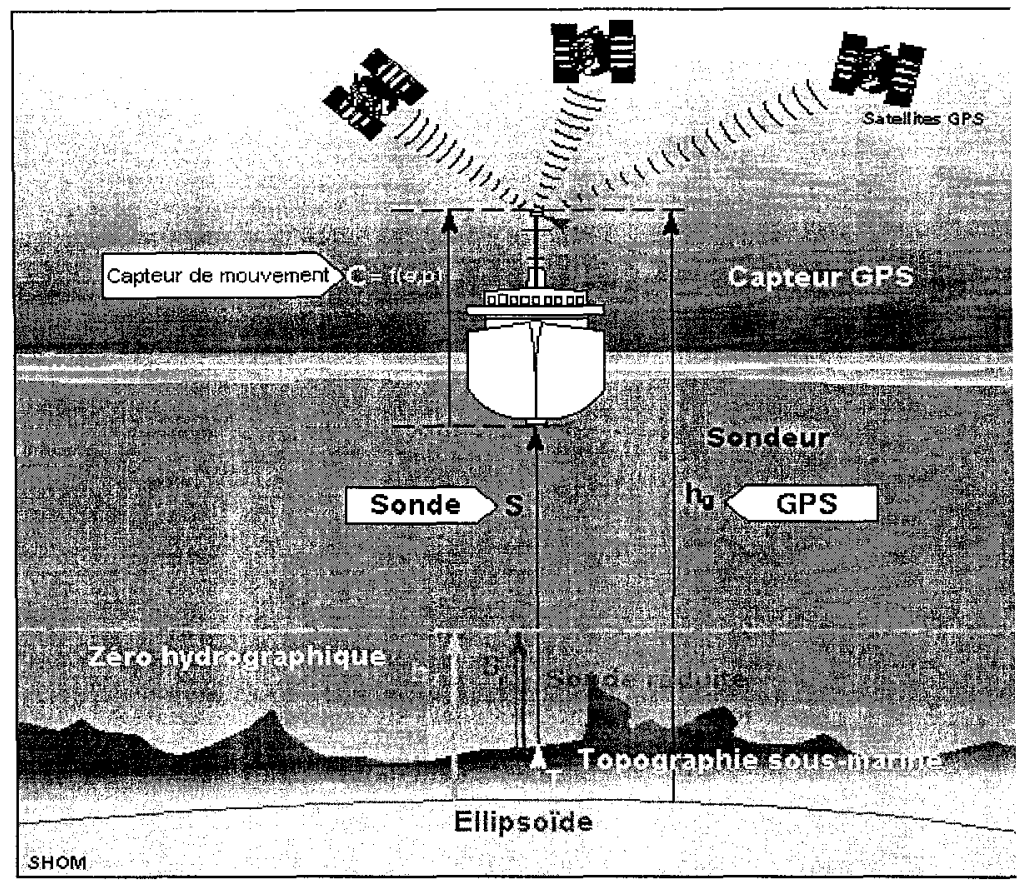

Figure 7. Réduction des sondages

Figure 7. Sounding reduction

La précision des réductions aux sondages dépend:

-de la précision de la surface de référence verticale (zéro hydrographique), -du positionnement relatif du sondeur à l'antenne GPS.

-du positionnement vertical du GPS cinématique par rapport à l'ITRS, -des erreurs propres aux mesures de profondeur.

Le tableau 3 présente l'évaluation des erreurs associées.

\begin{tabular}{|c|c|c|c|}
\hline & $\mathrm{d}<25 \mathrm{~km}$ (côte) & $25<\mathrm{d}<50 \mathrm{~km}$ & $\mathrm{~d}>50 \mathrm{~km}$ \\
\hline Zéro & $10-25 \mathrm{~cm}$ & $10 \mathrm{~cm}$ & $7 \mathrm{~cm}$ \\
\hline GPS Sondeur & $3 \mathrm{~cm}$ & $3 \mathrm{~cm}$ & $3 \mathrm{~cm}$ \\
\hline GPS cinématique & $5 \mathrm{~cm}$ & $9 \mathrm{~cm}$ & $11 \mathrm{~cm}$ \\
\hline TOTAL & $12-26 \mathrm{~cm}$ & $14 \mathrm{~cm}$ & $13 \mathrm{~cm}$ \\
\hline
\end{tabular}

Tableau 3: Evaluation des erreurs

Table 3: Evaluation and error propagation 
Les erreurs de mesures de profondeurs du sondeur étant de $15 \mathrm{~cm}$ environ, on atteint une précision meilleure que $30 \mathrm{~cm}$ pour les mesures de profondeurs par rapport au niveau des plus basses mers.

\section{$\underline{4 \text { Conclusion }}$}

L'approche spatiale présentée ici montre une définition et une réalisation du zéro hydrographique qui satisfont les recommandations internationales de l'OHI et de l'AIG et permet une certaine amélioration de :

- la sécurité pour la navigation,

- la cohérence le long de la limite terre-mer,

- la facilité de réalisation, de maintenance et d'accès (GPS...),

- la stabilité (invariable selon le temps) et la précision (quelques centimètres),

- l'impact sur la gestion des bases de données,

- la cohérence globale et une conséquente unification des zéros hydrographiques.

Cela permet aussi de faciliter le positionnement et la réduction des sondages et d'aboutir à une meilleure précision de la profondeur finale: en effet l'avantage majeur de cette approche est d'éviter le recours à la modélisation de la marée au large (longue et lourde à mettre en œuvre) et à l'estimation des effets de la météorologie (surcotes - décotes transportées de l'observatoire le plus proche).

Nous espérons dans le futur que le nouveau calcul de la SMO avec 7 ans de données TOPEX/POSEIDON (une couverture temporelle des océans plus complète) nous fournira une amélioration notable de la précision proche des côtes. Evidemment notre approche sera améliorée avec un nombre plus important de marégraphes reliés à des GPS.

\section{Références}

[1] Boucher C., Z. Altamimi and P. Sillard [1998] : "Results and analysis of the ITRF96". IERS technical note 24, May 1998

[2] Hernandez F. et P.Schaeffer [1999] : "Surface moyenne océanique CLS_SHOM98.2. Présentation et validation". Rapport 1, CLS/DOS/NT/99.141, avril 1999.

[3] Pineau L. [1999] : "Amélioration du modèle de prédiction de marée en Manche". Rapport 282 EPSHOM/CH/GG/ET/NP du 28.05.99.

[4] SHOM [1994] : "La lettre du SHOM aux navigateurs", n¹1, décembre 1994, pp 6-8

[5] Simon B. [1990] "Calcul de la marée au large pour la correction des sondages", Revue Hydrographique Internationale, vol LXVII (2), juillet 1990.

[6] Wöppelmann G., S. Allain, P. Bahurel, S. Lannuzel et B. Simon [1999] : "Zéro hydrographique : vers une détermination globale". Revue XYZ, Nr. 79, 2e trimestre 1999, pp. 27-34. 